Article

\title{
Prediction of the Epidemic Peak of Coronavirus Disease in Japan, 2020
}

\section{Toshikazu Kuniya $\mathbb{D}$}

Graduate School of System Informatics, Kobe University, 1-1 Rokkodai-cho, Nada-ku, Kobe 657-8501, Japan; tkuniya@port.kobe-u.ac.jp

Received: 24 February 2020; Accepted: 10 March 2020; Published: 13 March 2020

\begin{abstract}
The first case of coronavirus disease 2019 (COVID-19) in Japan was reported on 15 January 2020 and the number of reported cases has increased day by day. The purpose of this study is to give a prediction of the epidemic peak for COVID-19 in Japan by using the real-time data from 15 January to 29 February 2020. Taking into account the uncertainty due to the incomplete identification of infective population, we apply the well-known SEIR compartmental model for the prediction. By using a least-square-based method with Poisson noise, we estimate that the basic reproduction number for the epidemic in Japan is $R_{0}=2.6(95 \% \mathrm{CI}, 2.4-2.8)$ and the epidemic peak could possibly reach the early-middle summer. In addition, we obtain the following epidemiological insights: (1) the essential epidemic size is less likely to be affected by the rate of identification of the actual infective population; (2) the intervention has a positive effect on the delay of the epidemic peak; (3) intervention over a relatively long period is needed to effectively reduce the final epidemic size.
\end{abstract}

Keywords: COVID-19; SEIR compartmental model; basic reproduction number

\section{Introduction}

In December 2019, the first case of respiratory disease caused by a novel coronavirus was identified in Wuhan City, Hubei Province, China. The outbreak of the disease is ongoing worldwide and the World Health Organization named it coronavirus disease 2019 (COVID-19) on 11 February 2020 [1]. In Japan, the first case was reported on 15 January 2020 and the number of reported laboratory-confirmed COVID-19 cases per week has increased day by day (see Table 1).

Table 1. Number of newly reported COVID-19 cases in Japan until 1 March 2020 [2].

\begin{tabular}{lcc}
\hline Week & Number of Newly Reported Cases & Number of Accumulated Cases \\
\hline 12 January-18 January & 1 & 1 \\
19 January-25 January & 2 & 3 \\
26 January-1 February & 14 & 17 \\
2 February-8 February & 8 & 25 \\
9 February-16 February & 28 & 53 \\
17 February-23 February & 79 & 132 \\
24 February-1 March & 107 & 239 \\
\hline
\end{tabular}

As seen in Table 1, the number of newly reported cases per week has increased and a serious outbreak in Japan is a realistic outcome. One of the greatest public concerns is whether the epidemic continues until summer so that it affects the Summer Olympics, which is planned to be held in Tokyo. The purpose of this study is to give a prediction of the epidemic peak of COVID-19 in Japan, which might help us to act appropriately to reduce the epidemic risk. 
The epidemic data as shown in Table 1 would have mainly twofold uncertainty. The first one is due to the fact that asymptomatic infected people could spread the infection [3]. The second one is due to the lack of opportunity for the diagnostic test as sufficiently simple diagnostic test kits have not been developed yet and the diagnosis in the early stage in Japan was mainly restricted to people who visited Wuhan [4]. In this study, taking into account such uncertainty, we apply a simple and well-known mathematical model for the prediction. More precisely, we assume that only $p(0<p \leq 1)$ fraction of infective individuals can be identified by diagnosis.

\section{Methods}

\subsection{Model}

We apply the following well-known SEIR compartmental model (see, e.g., [5]) for the prediction.

$$
\left\{\begin{array}{l}
S^{\prime}(t)=-\beta S(t) I(t), \quad E^{\prime}(t)=\beta S(t) I(t)-\varepsilon E(t), \\
I^{\prime}(t)=\varepsilon E(t)-\gamma I(t), \quad R^{\prime}(t)=\gamma I(t), \quad t>0,
\end{array}\right.
$$

where $S(t), E(t), I(t)$ and $R(t)$ denote the susceptible, exposed, infective and removed populations at time $t$, respectively. $\beta, \varepsilon$ and $\gamma$ denote the infection rate, the onset rate and the removal rate, respectively. Note that $1 / \varepsilon$ and $1 / \gamma$ imply the average incubation period and the average infectious period, respectively. Let the unit time be 1 day. Based on the previous studies [6,7], we fix $1 / \varepsilon=5$, and thus, $\varepsilon=0.2$ and $\gamma=0.1$, respectively. We fix $S+E+I+R$ to be 1 so that each population implies the proportion to the total population. We assume that one infective person is identified at time $t=0$ among total $N=1.26 \times 10^{8}$ number of people in Japan [8]. That is, $Y(0)=p I(0) \times 1.26 \times 10^{8}=1$, where

$$
Y(t)=p I(t) \times 1.26 \times 10^{8}
$$

denotes the number of infective individuals who are identified at time $t$. Thus, we obtain $I(0)=$ $1 /\left(p \times 1.26 \times 10^{8}\right)$. We assume that there is no exposed and removed populations at $t=0$, that is, $E(0)=R(0)=0$, and hence,

$$
S(0)=1-E(0)-I(0)-R(0)=1-\frac{1}{p \times 1.26 \times 10^{8}} .
$$

It was estimated in [9] that 77 cases were confirmed among the possible 940 infected population in February in Hokkaido, Japan. Based on this report, we assume that $p$ ranges from 0.01 to 0.1 . The basic reproduction number $R_{0}$, which means the expected value of secondary cases produced by one infective individual [10], is calculated as the maximum eigenvalue of the next generation matrix $F V^{-1}[11]$, where

$$
F=\left[\begin{array}{cc}
0 & \beta S(0) \\
0 & 0
\end{array}\right], \quad V=\left[\begin{array}{cc}
\varepsilon & 0 \\
-\varepsilon & \gamma
\end{array}\right]
$$

Thus, we obtain

$$
R_{0}=\frac{\beta S(0)}{\gamma}=\frac{\beta}{\gamma}\left(1-\frac{1}{p \times 1.26 \times 10^{8}}\right)
$$

\subsection{Sensitivity of the Basic Reproduction Number}

It is obvious that the basic reproduction number $R_{0}$ is independent from the onset rate $\varepsilon$. The sensitivity of $R_{0}$ to other parameters $\beta, \gamma$ and $p$ are calculated as follows:

$$
A_{\beta}=\frac{\beta}{R_{0}} \frac{\partial R_{0}}{\partial \beta}=1, \quad A_{\gamma}=\frac{\gamma}{R_{0}} \frac{\partial R_{0}}{\partial \gamma}=-1, \quad A_{p}=\frac{p}{R_{0}} \frac{\partial R_{0}}{\partial p}=\frac{1}{p \times 1.26 \times 10^{8}-1},
$$


where $A_{\beta}, A_{\gamma}$ and $A_{p}$ denote the normalized sensitivity indexes with respect to $\beta, \gamma$ and $p$, respectively. We see from Equation (3) that the $k$ time's increase in $\beta$ (resp. $\gamma$ ) results in the $k$ (resp. $k^{-1}$ ) time's increase in $R_{0}$. In particular, we see from the third equation in Equation (3) that $A_{p} \approx 0$ if $p \geq 1.0 \times 10^{-6}$. This implies that the identification rate $p$ in a realistic range almost does not affect the size of $R_{0}$.

\subsection{Estimation of the Infection Rate}

Let $y(t), t=0,1, \ldots, 45$ be the number of daily reported cases of COVID-19 in Japan from 15 January $(t=0)$ to 29 February $(t=45) 2020$. We perform the following least-square-based procedure with Poisson noise to estimate the infection rate $\beta$.

\section{Description 1.}

(P1) Fix $\beta>0$ and calculate the numerical value of $Y(t), t=0,1, \ldots, 45$ by using model Equation (1).

(P2) Calculate

$$
\tilde{Y}(t)=Y(t)+\sqrt{Y(t)} \epsilon(t)=Y(t)+(\text { Poisson noise }), \quad t=0,1, \ldots, 45,
$$

where $\epsilon(t), t=0,1, \ldots, 45$ denote random variables from a normal distribution with mean zero and variance 1 [12].

(P3) Calculate $J(\beta)=\sum_{t=0}^{45}[y(t)-\tilde{Y}(t)]^{2}$.

(P4) Run (P1)-(P3) for $0.2 \leq \beta \leq 0.4$ and find $\beta^{*}$ such that $J\left(\beta^{*}\right)=\min _{0.2 \leq \beta \leq 0.4} J(\beta)$.

(P5) Repeat (P1)-(P4) 10, 000 times and obtain the distribution of $\beta^{*}$.

(P6) Approximate the distribution of $\beta^{*}$ by a normal distribution and obtain a $95 \%$ confidence interval.

Note that for the reason stated above, the value of $0.01 \leq p \leq 0.1$ does not affect this estimation procedure. By (P1)-(P6), we obtain a normal distribution with mean 0.26 and standard derivation 0.01 . Thus, we obtain an estimation of $\beta$ as 0.26 (95\%CI, 0.24-0.28) (see Figure 1). Moreover, by Equation (2), we obtain an estimation of $R_{0}$ as $2.6(95 \% \mathrm{CI}, 2.4-2.8)$ (see Table 2$)$.

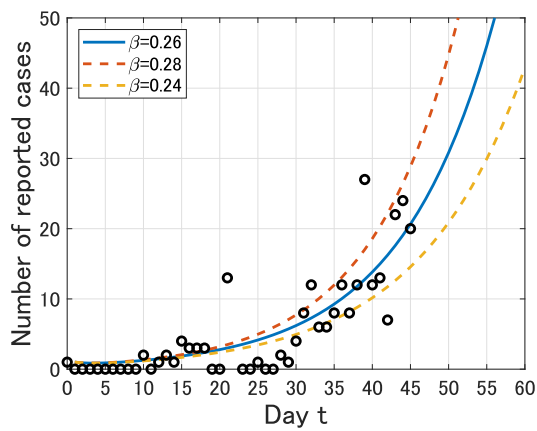

Figure 1. Comparison of $Y(t)$ with the estimated infection rate $\beta$ and the number of daily reported cases of COVID-19 in Japan from 15 January $(t=0)$ to 29 February $(t=45)$.

Table 2. Parameter values for model Equation (1).

\begin{tabular}{clcc}
\hline Parameter & Description & Value & Reference \\
\hline$\beta$ & Infection rate & $0.26(95 \% \mathrm{CI}, 0.24-0.28)$ & Estimated \\
$R_{0}$ & Basic reproduction number & $2.6(95 \% \mathrm{CI}, 2.4-2.8)$ & Estimated \\
$\varepsilon$ & Onset rate & 0.2 & {$[6]$} \\
$\gamma$ & Removal rate & 0.1 & {$[7]$} \\
$N$ & Total population in Japan & $1.26 \times 10^{8}$ & {$[8]$} \\
$p$ & Identification rate & $0.01-0.1$ & {$[9]$} \\
\hline
\end{tabular}




\section{Results}

\subsection{Peak Prediction}

We define the epidemic peak $t^{*}$ by the time such that $Y$ attains its maximum in 1 year, that is, $Y\left(t^{*}\right)=\max _{0 \leq t \leq 365} Y(t)$. We first set $p=0.1$. In this case, we obtain the following figure on the long time behavior of $Y(t)$ for $\beta=0.28,0.26$ and 0.24 .

We see from Figure 2 that the estimated epidemic peak is $t^{*}=208$ (95\%CI, 191-229). That is, starting from 15 January $(t=0)$, the estimated epidemic peak is 10 August $(t=208)$ and the uncertainty range is from 24 July $(t=191)$ to 31 August $(t=229)$.

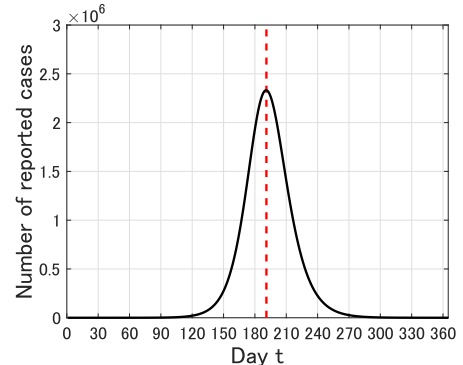

(a) $\beta=0.28\left(t^{*}=191\right)$

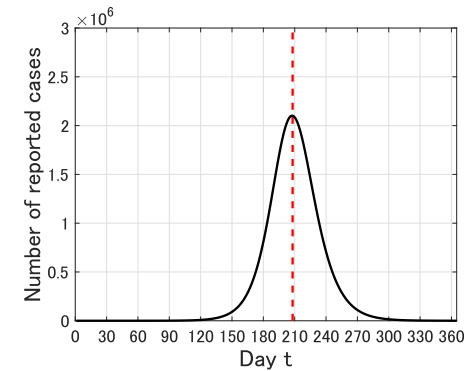

(b) $\beta=0.26\left(t^{*}=208\right)$

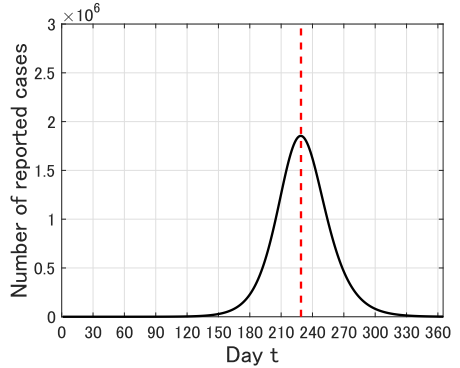

(c) $\beta=0.24\left(t^{*}=229\right)$

Figure 2. Time variation of the number $Y(t)$ of infective individuals who are identified at time $t$ $(0 \leq t \leq 365)$ for $p=0.1$. The dot lines represent the epidemic peak $t^{*}$.

We next set $p=0.01$. In this case, we obtain the following figure.

We see from Figure 3 that the estimated epidemic peak is $t^{*}=179$ (95\%CI, 165-197). That is, starting from January $15(t=0)$, the estimated epidemic peak is July $12(t=179)$ and the uncertainty range is from June $28(t=165)$ to July $30(t=197)$. In contrast to $R_{0}$, the epidemic peak and the (apparent) epidemic size are sensitive to the identification rate $p$. Note that the essential epidemic size, which is characterized by $R_{0}$, is almost the same in both of $p=0.1$ and $p=0.01$.

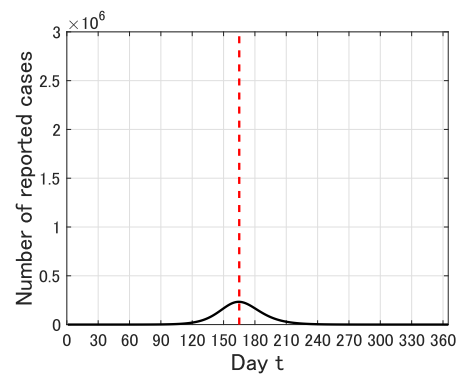

(a) $\beta=0.28\left(t^{*}=165\right)$

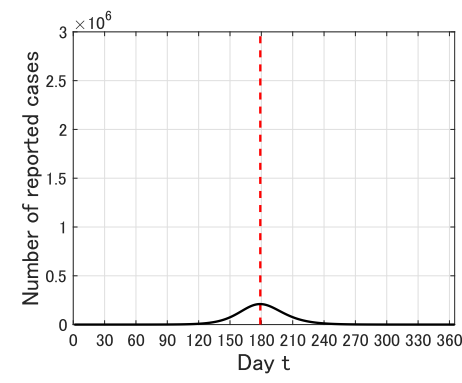

(b) $\beta=0.26\left(t^{*}=179\right)$

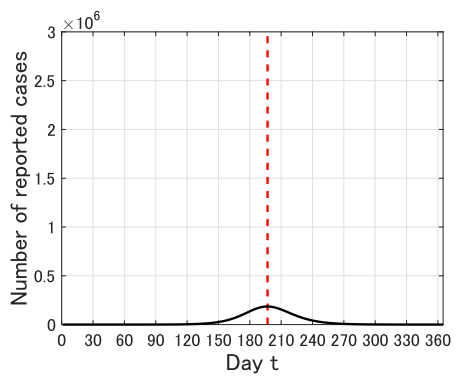

(c) $\beta=0.24\left(t^{*}=197\right)$

Figure 3. Time variation of the number $Y(t)$ of infective individuals who are identified at time $t$ $(0 \leq t \leq 365)$ for $p=0.01$. The dot lines represent the epidemic peak $t^{*}$.

\subsection{Possible Effect of Intervention}

We next discuss the effect of intervention. In Japan, school closure has started in almost all prefectures from the beginning of March [13] and many social events have been cancelled off to reduce the contact risk. However, the exact effect of such social efforts is unclear and might be limited as the proportion of young people to the whole infected people of COVID-19 seems not so high (2\% of 72,314 reported cases in China [14]). In this simulation, we assume that such social efforts successfully reduce the infection rate $\beta=0.26$ to $75 \%$ during a period from 1 March $(t=46)$ to a planned day $(t=T \geq 47)$. In what follows, we fix $p=0.01$. 
First, we set $T=77$, that is, the intervention is carried out for 1 month (from 1 March to 1 April). In this case, the epidemic peak $t^{*}$ is delayed from 179 (12 July) to 190 (23 July). However, the epidemic size is almost the same. On the other hand, if $T=220$, that is, the intervention is carried out for 6 months (from 1 March to 1 September), then the epidemic peak $t^{*}$ is delayed from 179 (12 July) to 243 (14 September) and the epidemic size is effectively reduced (see Figure 4).

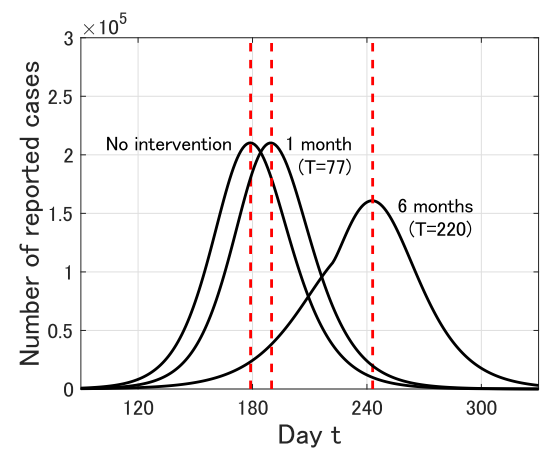

Figure 4. Time variation of the number $Y(t)$ of infective individuals who are identified at time $t$ $(0 \leq t \leq 365)$ for $p=0.01$ and no intervention, 1 month intervention $(T=77)$ and 6 months intervention $(T=220)$. The dot lines represent the epidemic peak.

More precisely, we see from Figure $5 \mathrm{a}$ that the epidemic peak $t^{*}$ is delayed almost linearly for $47 \leq T \leq 239$ and fixed to $t^{*}=237$ for $T \geq 240$.

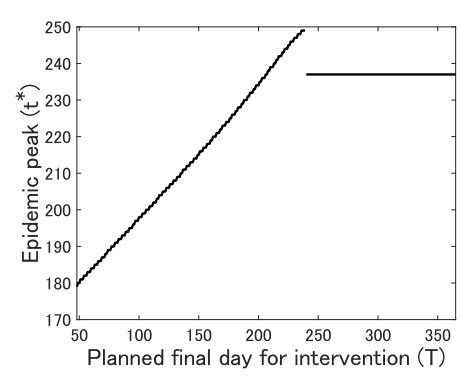

(a)

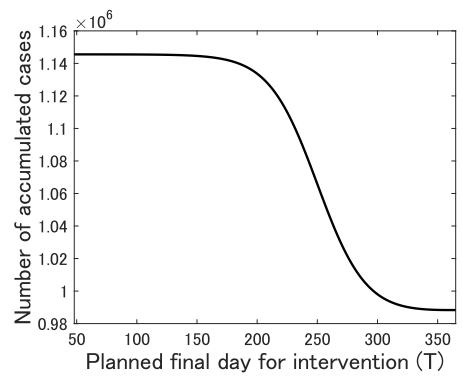

(b)

Figure 5. The relation between the planned final day for intervention $T$ and (a) the epidemic peak $t^{*}$;

(b) the number of accumulated cases at time $t=365$ : $p R(365) \times 1.26 \times 10^{8}$.

This implies that the intervention has a positive effect on the delay of the epidemic peak, which would contribute to improve the medical environment utilizing the extra time period. On the other hand, we see from Figure $5 \mathrm{~b}$ that the number of accumulated cases at $t=365$, which is calculated as $p R(365) \times 1.26 \times 10^{8}$, is monotonically decreasing and converges to $0.99 \times 10^{6}$ as $T$ increases. However, it almost does not change for small $T \leq 180$. This implies that the intervention over a relatively long duration is required to effectively reduce the final epidemic size.

\section{Discussion}

In this study, by applying the SEIR compartmental model to the daily reported cases of COVID-19 in Japan from 15 January to 29 February, we have estimated that the basic reproduction number $R_{0}$ is 2.6 (95\%CI, 2.4-2.8) and the epidemic peak could possibly reach the early-middle summer. Of course, this kind of long range peak prediction would contain the essential uncertainty due to the possibility of some big changes in the social and natural (climate) situations. Nevertheless, our result suggests that the epidemic of COVID-19 in Japan would not end so quickly. This might be consistent with the WHO's statement on 6 March 2020 that it is a false hope that COVID-19 will disappear in the summer like the flu [15]. 
The estimated value of the basic reproduction number $R_{0}$ in this study is not so different from early estimations: 2.6 (95\%CI, 1.5-3.5) [16], 2.90 (95\%CI, 2.32-3.63) [17], 3.11 (95\%CI, 2.39-4.13) [18], 3.58 (95\%CI, 2.89-4.39) [19] and 3.28 (average of estimations in 12 studies) [20]. In addition, in this study, we have obtained the following epidemiological insights:

- The essential epidemic size, which is characterized by $R_{0}$, would not be affected by the identification rate $p$ in a realistic parameter range 0.01-0.1, in particular, $p \geq 1.0 \times 10^{-6}$.

- The intervention exactly has a positive effect on the delay of the epidemic peak, which would contribute to improve the medical environment utilizing the extra time period.

- Intervention over a relatively long period is needed to effectively reduce the final epidemic size.

The first statement implies that underestimation of the actual infective population would not contribute to the reduction of the essential epidemic risk. Correct information based on an adequate diagnosis system would be desired for people to act appropriately.

Funding: This research was funded by the Japan Society for the Promotion of Science (JSPS) KAKENHI grant number 19K14594.

Acknowledgments: The author would like to thank the associate editor and the anonymous reviewers for their helpful comments that allowed me to improve the manuscript.

Conflicts of Interest: The author declares no conflict of interest.

\section{References}

1. Centers for Disease Control and Prevention. Coronavirus Disease 2019 (COVID-19). Available online: https: / /www.cdc.gov/ coronavirus/2019-ncov/index.html (accessed on 23 February 2020).

2. World Health Organization. Coronavirus Disease 2019 (COVID-19) Situation Reports. Available online: https: / / www.who.int/emergencies/diseases/novel-coronavirus-2019/situation-reports/ (accessed on 5 March 2020).

3. Rothe, C.; Schunk, M.; Sothmann, P.; Bretzel, G.; Froeschl, G.; Wallrauch, C.; Zimmer, T.; Thiel, V.; Jankem, C.; Guggemos, W.; et al. Transmission of 2019-nCoV Infection from an Asymptomatic Contact in Germany. New Eng. J. Med. 2020. [CrossRef] [PubMed]

4. NHK World Japan. Japan Sets up Emergency Measures for Coronavirus. Available online: https://www3. nhk.or.jp/nhkworld/en/news/20200213_07/ (accessed on 17 February 2020).

5. Inaba, H. Age-Structured Populatin Dynamics in Demography and Epidemiology; Springer: Berlin/Heidelberg, Germany, 2017.

6. $\quad$ Linton, N.M.; Kobayashi, T.; Yang, Y.; Hayashi, K.; Akhmetzhanov, A.R.; Jung, S.; Yuan, B.; Kinoshita, R.; Nishiura, H. Incubation period and other epidemiological characteristics of 2019 novel coronavirus infections with right truncation: A statistical analysis of publicly available case data. J. Clin. Med. 2020, 9, 538. [CrossRef] [PubMed]

7. Sun, H.; Qiu, Y.; Yan, H.; Huang, Y.; Zhu, Y.; Chen, S.X. Tracking and predicting COVID-19 epidemic in China mainland. medRxive 2020. [CrossRef]

8. Statistics Bureau Japan. Population Estimates Monthly Report January. Available online: https://web. archive.org/web/20190623053923/http:/ /www.stat.go.jp/english/data/jinsui/tsuki/index.html (accessed on 5 March 2020).

9. Bloomberg. Japan's Hokkaido may Have 940 Infected, Researcher Says. Available online: https://www. bloomberg.com/news/articles/2020-03-03/japan-s-hokkaido-could-have-up-to-940-infected-researcher-says (accessed on 5 March 2020).

10. Diekmann, O.; Heesterbeek, J.A.P.; Metz, J.A.J. On the definition and the computation of the basic reproduction ratio $R_{0}$ in models for infectious diseases in heterogeneous populations. J. Math. Biol. 1990, 28, 365-382. [CrossRef] [PubMed]

11. van den Driessche, P.; Watmough, J. Reproduction numbers and sub-threshold endemic equilibria for compartmental models of disease transmission. Math. Biosci. 2002, 180, 29-48. [CrossRef]

12. Capaldi, A.; Behrend, S.; Berman, B.; Smith, J.; Wright, J.; Lloyd, A.L. Parameter estimation and uncertainty quantification for an epidemic model. Math. Biosci. Eng. 2012, 9, 553-576. [PubMed] 
13. The Japan Times. Nearly All Prefectures in Japan Shut Schools Amid Coronavirus Outbreak. Available online: https: / www.japantimes.co.jp/news/2020/03/02/national/japan-prefectures-shut-schools-covid19/\#.XmIKE0BuKUk (accessed on 6 March 2020).

14. Wu, Z.; McGoogan, J.M. Characteristics of and imoprtant lessons from the coronavirus disease 2019 (COVID-19) outbreak in China. J. Am. Med. Assoc. 2020. [CrossRef] [PubMed]

15. CNBC. It's a 'false hope' Coronavirus will Disappear in the Summer like the Flu, WHO Says. Available online: https:/ / www.cnbc.com/2020/03/06/its-a-false-hope-coronavirus-will-disappear-in-the-summerlike-the-flu-who-says.html (accessed on 8 March 2020).

16. Imai, N.; Cori, A.; Dorigatti, I.; Baguelin, M.; Connelly, C.A.; Riley, S.; Ferguson, N.M. Report 3: Transmissibility of 2019-nCoV; Imperial College London: London, UK, 2020.

17. Liu, T.; Hu, J.; Kang, M.; Lin, L.; Zhong, H.; Xiao, J.; He, G.; Song, T.; Huang, Q.; Rong, Z.; et al. Transmission dynamics of 2019 novel coronavirus (2019-nCoV). bioRxive 2020. [CrossRef]

18. Read, J.M.; Bridgen, J.R.E.; Cummings, D.A.T.; Ho, A.; Jewell C.P. Novel coronavirus 2019-nCoV: Ealry estimation of epidemiological parameters and epidemic predictions. medRxive 2020. [CrossRef]

19. Zhao, S.; Lin, Q.; Ran, J.; Musa, S.S.; Yang, G.; Wang, W.; Lou, Y.; Gao, D.; Yang, L.; He, D.; et al. Preliminary estimation of the basic reproduction number of novel coronavirus (2019-nCoV) in China, from 2019 to 2020: A data-driven analysis in the early phase of the outbreak. Int. J. Infect. Dis. 2020, 92, 214-217. [CrossRef] [PubMed]

20. Liu, Y.; Gayle, A.A.; Wilder-Smith, A.; Rocklöv, J. The reproductive number of COVID-19 is higher compared to SARS coronavirus. J. Travel. Med. 2020. [CrossRef] [PubMed]

(C) 2020 by the authors. Licensee MDPI, Basel, Switzerland. This article is an open access article distributed under the terms and conditions of the Creative Commons Attribution (CC BY) license (http://creativecommons.org/licenses/by/4.0/). 